\title{
THE THERMODYNAMIC DESCRIPTION OF PHASE TRANSITIONS*
}

\author{
Michael H. COOPERSMith \\ Department of Physics and Center for Advanced Studies, \\ University of Virginia, McCormick Road, Charlottesville, Va 22903, U.S.A.
}

\begin{abstract}
The historical fact of the ability of a system to avoid showing a phase transition when taken along a suitable thermodynamic path is examined and a way offered of looking at the 'critical point' problem in keeping with various prevailing viewpoints. The semi-phenomenological view is given, followed by exact results, perturbation expansions, a description of equilibrium phenomena and illustrations which involve the Riemann surface but omit the property of convexity.
\end{abstract}

\section{INTRODUCTION}

Despite the recent heroic attempts to construct microscopic models whose exact solutions show phase transitions, there remains a peculiar gap between the equations of state which arise from these models and the experimentally defined relationships among the thermodynamic parameters. This gap is all the more striking due to the fact that good quantitative agreement with experiment can be obtained for a wide range of parameters but there is a decided lack of qualitative agreement near just those values of the parameters which appear to be most interesting. These are the values near a critical point of the system.

That the neighbourhood of a critical point is indeed the most interesting place to look at a phase transition is not only a latter day phenomenon but also an historical fact. In looking over the literature pertaining to changes of state, one is immediately struck by the concern of early workers in the field with the fact that, above certain critical values of the parameters, no change of state of the system can be observed. Thus, as early as 1822, one Baron de la Tour ${ }^{1}$ observed that various gases including carbon dioxide and water vapour exist in only one phase when the temperature is above the value now commonly known as the critical temperature. Later experiments confirmed these results more quantitatively ${ }^{2}$ but de la Tour's work had already uncovered the basic phenomenon described by van der Waals ${ }^{3}$ in his now classic equation of state. This 'continuity of liquid and vapour states' (as van der Walls referred to it) or the ability of a system to avoid showing a phase transition when taken along a suitable thermodynamic path represents the most outstanding feature of the critical region and may, in fact, be thought of as the essence of a critical point. We offer here a way of

\footnotetext{
* As the author was unable to be present at the Conference, this paper was read by title only.
} 
looking at the problem which ties together various prevailing viewpoints. Much of this material is excerpted from an article of the same title by the author ${ }^{4}$.

\section{THE SEMI-PHENOMENOLOGICAL VIEW}

The idea of a semi-phenomenological explanation for the continuity of states originated, as already mentioned, with van der Waals, was continued by Weiss ${ }^{5}$ for an explanation of the Curie point of ferromagnetism, and reached its culmination in the generalization of the van der Waals equation of state proposed by Widom ${ }^{6}$ a few years ago. The prefix 'semi' is justified because one can concoct microscopic models which yield the van der Waals and Weiss equations of state in some approximation. These models have been discussed extensively in the literature ${ }^{7}$ and will not be taken up here. We shall only note that the physical basis of these equations of state comes about from the competition between energy and entropy in the Helmholtz free energy, $F=E-T S$, the one arising from the weak, long range attraction between the particles and the other from the strong, short range repulsion (generally taken as a hard core). Widom's homogeneous equations of state do not have such a simple explanation ${ }^{8}$ but if we accept the idea of homogeneity as at least an approximation to some general physical principle, then these equations too may be described as semi-phenomenological.

In order to put this viewpoint in its proper perspective, we refer to an analysis of free energies (i.e. thermodynamic potentials) which show the phenomenon of the critical point as given by the author ${ }^{9}$. This treatment relies on the sole assumption of analytical continuation of the thermodynamic potential in both its variables. The main results are:

(1) In the neighbourhood of the Curie (critical) point, the Weiss theory free energy $F(H, T)$ can be represented by an algebraic function of third degree. The chemical potential corresponding to the van der Waals equation of state in the neighbourhood of the critical point has the same form when regarded as a function of reduced pressure and temperature after a transformation which makes the reduced vapour pressure equal to zero for all temperatures. (We use the word 'reduced' to denote the difference between a variable and its critical value.)

(2) Magnetic free energies which are algebraic functions homogeneous in the applied field and reduced temperature form a special case of the analogue of Widom's homogeneous functions.

The assumption that a thermodynamic potential is an algebraic function of its natural variables is clearly an approximation since (a) the critical exponents must be rational and (b) no logarithmic behaviour can be obtained. However, we shall see that algebraic functions act as a framework upon which to hang the more general description which will include logarithmic behaviour as well as other singularities.

\section{EXACT RESULTS}

It seems increasingly clear that a simple closed analytical expression for 
the full ferromagnetic problem with applied field or the related problem of a fluid is by its very nature impossible to obtain. The reason boils down to a question of representation of a function. In ref. 9 it is seen that even the simplest algebraic functions which show the characteristic behaviour of a critical point cannot be represented in closed form but are defined as implicit functions by a polynomial equation. The representation is then realized by expanding the individual terms in the polynomial about any point of interest, thus obtaining a representation of the function about that point. This representation is in the form of an ordinary Taylor expansion if the point is non-singular and a Taylor expansion in the variable $\zeta=z^{1 / n}$ if the point is a singular one. Here $z$ is the original variable, $n$ is the degree of the defining polynomial (more accurately, the order of the singular point) and the expansion may start with a negative but finite power of $\zeta$. In the neighbourhood of a critical point, we are dealing with a function of two variables and the points of interest lie on either the coexistence curve or the critical isotherm. The critical point itself is excluded. For the magnetic case, the expansions are of the form

for the first case and

$$
F(H, T)=\sum_{n=0}^{\infty} a_{n}\left(T-T_{c}\right) H^{n}
$$

$$
F(H, T)=\sum_{n=0}^{\infty} b_{n}(H)\left(T-T_{c}\right)^{n}
$$

for the second. The coefficients $a$ and $b$ are themselves expandable in the form required for a singular (branch) point in one variable, so that we have

and

$$
a_{n}(z)=\sum_{i=r_{n}}^{\infty} A_{n i} z^{i / t_{n}}
$$

$$
b_{n}(z)=\sum_{i=s_{n}}^{\infty} B_{n i} z^{i / u_{n}}
$$

where $r$ and $s$ are positive or negative integers and $t$ and $u$ are the orders of the branch points. The numbers $r_{n} / t_{n}$ and $s_{n} / u_{n}$ (referred to as $\alpha_{n}$ and $\beta_{n}$ in ref. 9) are the leading exponents and are commonly known as the critical exponents for the $n$th derivative of the free energy with respect to $H$ and $T$ respectively. For the two-dimensional Ising model, only the asymptotic forms of the leading terms of $a_{0}^{ \pm}\left(T-T_{c}\right)$ and $a_{1}^{-}\left(T-T_{c}\right)$ have been found analytically. (The plus and minus signs refer to whether $T$ is greater than or less than $T_{c}$, that is, whether we are above or below the critical point.) Onsager's well known solution ${ }^{10}$ gives $a_{0}^{+}\left(T-T_{c}\right)$ and $a_{0}^{-}\left(T-T_{c}\right)$ both asymptotically equal to $\left(T-T_{c}\right)^{2} \ln \left(\left|T-T_{c}\right|\right)$ while the published solution of Yang ${ }^{11}$ for the spontaneous magnetization gives the asymptotic form of $a_{1}^{-}\left(T-T_{c}\right)$ as $\left(T_{c}-T\right)^{\frac{1}{d}}$. None of the other as and $b \mathrm{~s}$ are known even asymptoticallyt. Nevertheless, the mean field theories together with some recent work on perturbation expansions to be discussed in the next section contain what we believe to be the essential features of phase transitions.

$\dagger$ Various numerical calculations for the $a_{n} \mathrm{~s}$ and $b_{n} \mathrm{~s}$ up to $n=8$ have been made. See refs. 12 and 13. 


\section{PERTURBATION EXPANSIONS}

The expansion about the ideal gas pressure with the fugacity as the variable has been returned to recently by a number of authors, among them Andreev ${ }^{14}$, Langer ${ }^{15}$ and Fisher ${ }^{16}$. It is shown that if one considers an expansion in the fugacity of real droplets (as opposed to mathematical clusters), one is led to a series which represents a function which has an essential singularity on the real axis for all temperatures. Thus, the conjecture ${ }^{17}$ that there be a singularity at the condensation point (as opposed to the critical point) has been verified in a physically appealing approximation. Furthermore, Fisher ${ }^{16}$ has also analysed a one-dimensional model which shows these properties.

We consider the Riemann surface of an algebraic function of the type proposed in ref. 9. On a neighbourhood of this Riemann surface not containing a branch point, one may define a single valued function ${ }^{18}$. We now imagine a function defined on the Riemann surface which has an essential singularity at the crunode (see ref. 9) and is linear in a neighbourhood of the crunode. If the essential singularity be chosen so that its value and the values of all its derivatives approaching from the real axis are zero (the most common example is $\mathrm{e}^{-1 / z^{2}}$ ) then we will have a function of the type which has been found for the droplet model ${ }^{16}$. A specific example is the following. Let the algebraic function of two variables be denoted by $\mathrm{f}\left(z, T-T_{c}\right)$ where $z$ is the variable appropriate to the physical system (reduced chemical potential for a fluid, magnetic field for a ferromagnet). Let $\mathrm{g}(z)$ be a function which has an essential singularity of the type previously mentioned. The function $\mathrm{f}\left[\mathrm{g}(z)+z, T-T_{c}\right]$ then has all the properties ascribed by Fisher to the grand potential of his one-dimensional model. Furthermore, the line of essential singularities is continued above the transition temperature as in that model.

\section{DESCRIPTION OF EQUILIBRIUM PHENOMENA}

In the preceding sections, we have attempted to show the relationship between the currently accepted views of critical phenomena and the picture presented by the author in ref. 9. In that paper, explicit use was made of algebraic functions as a representation of that thermodynamic potential whose natural variables are intensive. But we have already seen in Section II that algebraic functions cannot provide all the observed behaviour. We now pose the following question. What class of functions can exhibit all the known behaviour and yet is elementary in the sense that it has already been studied?

Before discussing this problem we might remark that there is a natural analogue of this situation in the description of decay of physical states. That problem might be thought of in the same light as the present one. That is, we have available an elementary function (the decreasing exponential) whose properties are obviously well known and which describes the qualitative physical behaviour. Nevertheless, as in the present case, the connection 
between the underlying microscopic dynamics and the observed macroscopic behaviour has never been completely established $\dagger$.

What then are the elementary functions describing critical phenomena? We have already seen that algebraic functions alone are capable of describing many aspects of the two phase region. The main feature of algebraic functions of two variables which is most useful for the present situation is the breaking up of the Riemann surface into disjoint pieces when one of the variables has the particular value which corresponds physically to the critical value of the thermodynamic variable. This feature may be kept by working with functions defined on the Riemann surface of the appropriate algebraic function. Since the only missing aspect of the equilibrium situation is the possible logarithmic behaviour of the coefficients $a_{n}\left(T-T_{c}\right)$ defined in equation 1 , we need only assume that the functions defined on the Riemann surface are Abelian integrals or products of analytical functions and Abelian integrals. Such functions are elementary in the sense defined above. Since an Abelian integral is defined as the integral of a rational function of an algebraic function and its variable on the Riemann surface of that algebraic function, then its singularities include poles and logarithmic singularities, but no essential singularities ${ }^{\ddagger}$.

With these functions, we may now have $a_{0}\left(T-T_{c}\right) \sim\left(T-T_{c}\right) \ln \left(T-T_{c}\right)$ as in the two-dimensional Ising model Also, since the singularities of an Abelian integral (in one variable) are isolated, we preserve the property of analytical continuation in two variables from one side of the two phase region to the other. The same procedure may be used to define a function with a non-algebraic branch point on the Riemann surface of an algebraic function. This leads to irrational critical exponents. Finally, as in Section IV, we can add an essential singularity at the crunode by forming the function $F\left[\mathrm{~g}(z)+z, T-T_{c}\right]$ where $F(x, y)$ is an Abelian integral defined on the Riemann surface of an algebraic function of $x$ and $y$ and $\mathrm{g}(z)$ is a function with an essential singularity of the type $\exp \left(-1 / z^{2}\right)$ at the origin.

\section{ILLUSTRATIONS}

The following free energies serve to illustrate the method of description of the preceding section. Because of space limitations, we shall not analyse the Riemann surfaces but merely write down the defining equations for the algebraic functions and indicate briefly how the analysis should be done.

The first example is the familiar molecular field theory with the reduced thermodynamic potential $f$ given as a function of the applied field $H$ and reduced temperature $T-T_{c}=t$. Since $f$ is known to be homogeneous and the exponent $\delta$ is equal to three, we are dealing with a third order algebraic function. Its defining equation is

† An example of this is the decay of an arbitrary state to its thermodynamic equilibrium value. While various approximation schemes enable one to calculate the relaxation time (i.e. the normalization of the exponent governing the time dependence), its actual existence has never been rigorously demonstrated.

\$ Since we will not make any computational use of Abelian integrals here, we merely catalogue some of their properties. The interested reader is referred to G. A. Bliss, ref. 18 for the precise definition of Abelian integrals as well as theorems relating to them. 


$$
P(f ; H, t)=f^{3}+\frac{3}{2} t^{2} f^{2}+\left(\frac{9}{16} t^{4}+\frac{27}{8} H^{2} t\right) f+\frac{81}{64} H^{4}+\frac{9}{32} H^{2} t^{3}=0
$$

Computation of the discriminant of $P$ (the remainder when $P$ is divided by $\partial P / \partial f)$ shows that the branch points are located at $H= \pm \frac{2}{3}(-t)^{\frac{2}{3}}$ in agreement with the positions of the end points of the loops in the molecular field theory. The critical exponents are obtained by inserting the expansions of equations 1 and 2 into the defining equation 5 and equating coefficients of equal powers of $H$ or $t$. We find easily:

$$
\begin{array}{ll}
a_{0}^{-}=-\frac{3}{4}(-t)^{2} & \\
a_{1}^{-}= \pm 3^{\frac{1}{2}}(-t)^{\frac{1}{2}} & a_{0}^{+}=0 \\
a_{2}^{-}=-\frac{1}{4}(-t)^{-1} & a_{1}^{+}=0 \\
b_{0}=-\left(\frac{3}{4}\right)^{\frac{4}{3}} H^{\frac{4}{3}} & a_{2}^{+}=-\frac{1}{2} t \\
b_{1}=\left(\frac{3}{2}\right)^{\frac{3}{3}} H^{\frac{2}{3}} & \\
b_{2}=-\frac{1}{2} &
\end{array}
$$

where the plus and minus signs on the $a$ s refer to the sign of $t$, that is whether we are above or below the critical point. For $H=0$, factorization of $P$ into $f\left(f+\frac{3}{4} t^{2}\right)^{2}$ indicates that the Riemann surface of $f$ becomes disjoint on the coexistence curve and that there is a crunode at $H=0$.

The second example is the somewhat less physical three-dimensional ideal Bose gas. Following Gunton and Buckingham, we look at the free energy for fixed density and variable density of particles in the zero momentum state. Within a phase factor, the order parameter or Bose moment $\psi$ is the square root of the density of zero momentum particles and the conjugate variable $\zeta$ is the Bose field. The reduced thermodynamic potential $f$ as a function of $\zeta$ and $t$ is homogeneous and the exponent $\delta$ is equal to five so we are now dealing with a fifth order algebraic function. Its defining equation is

$$
\begin{array}{r}
P(f ; \zeta, t)=f^{5}-\frac{1}{6} t^{3} f^{4}+\frac{25}{12} t^{2} f^{3}-\frac{1}{3} t^{4} \zeta^{2} f^{2}+\frac{125}{108} t^{2} \zeta^{4} f+\left(\frac{5}{6}\right)^{5} \zeta^{6} \\
-\frac{1}{6} t^{5} \zeta^{4}=0
\end{array}
$$

The root of the discriminant locate the branch points at $\zeta= \pm\left(16 / 5^{\frac{5}{2}}\right)(-t)^{\frac{5}{2}}$ and $\zeta=0$. This sticking of two of the branch points at $\zeta=0$ shows that the expansion of equation 1 must be changed to

$$
f(\zeta, t)=\sum_{n=0}^{\infty} a_{n / 2}^{-}(-t) \zeta^{n / 2}
$$

for $t<0$. The as and $b$ s are found to be:

$$
\begin{array}{ll}
a_{0}^{-}=0 & a_{0}^{+}=\frac{1}{6} t^{3} \\
a_{\frac{1}{2}}^{-}=0 & a_{1}^{+}=0 \\
a_{1}^{-}= \pm(-t)^{\frac{1}{2}} & a_{2}^{+}=-\frac{1}{2} t^{2} \\
a_{\frac{3}{2}}^{-}= \pm \frac{1}{3}(-t)^{-\frac{3}{4}} & b_{0}=-\frac{5}{6} \zeta^{\frac{5}{3}} \\
a_{2}^{-}=\frac{1}{8}(-t)^{-2} & b_{1}=\frac{1}{2} \zeta^{\frac{4}{3}} \\
& b_{2}=\frac{1}{10} \zeta^{\frac{2}{3}}
\end{array}
$$


For $\zeta=0$, factorization of $P$ into $f^{4}\left(f-\frac{1}{6} t^{3}\right)$ again shows that there is a crunode at $\zeta=0$. However, the fact that $a_{3}^{-}$exists means that the analogue of the susceptibility is infinite at $\zeta=0$ for all $t<u$.

The branch points in the neighbourhood of the origin illustrate another aspect of the critical region which has become popular recently, mainly with the experimentalists. Since the two physical branches of $f$ which form the crunode for $t<0$ must be connected to the other non-physical branches, there must be a branch on each of them which approaches the origin of the $H$-plane as $t \rightarrow 0$. The trajectory of these branch points in the $t-H$ manifold is the spinodal line originally referred to by van der Waals ${ }^{19}$. Since the spinodal line lies on a non-physical (perhaps, metastable) sheet, it is unreachable by an equilibrium experiment. Nevertheless, by measuring a thermodynamic quantity along, say, an isotherm close to the critical isotherm, one may approach the branch point on the spinodal line and thus measure the associated exponent. The most obvious thing to measure along an isotherm is the magnetization as a function of $H^{20,21}$. In the molecular field theory, this behaves as

$$
M \sim[H-H(t)]^{\frac{1}{2}}
$$

where $H(t)$ is the trajectory of branch points. One may also approach the spinodal line along other curves; typically, for a liquid, $C_{V}$ may be measured along a non-critical isochore.

\section{CONCLUSION}

We have attempted to take some of the mystery out of the mathematical description of thermodynamic potentials near a critical point by showing that there are elementary functions which possess all the properties normally ascribed to these potentials in the vicinity of such a point. For the thermodynamic potential whose natural variables are naturally intensive, these properties may be summarized as follows. The thermodynamic potential is denoted by $F$ and its reduced natural variables by $z$ and $t$.

(1) $F$ is a single valued function of $z$ and $t$ with a singularity in $z$ and $t$ separately at $z=t=0$ (critical point).

(2) $(\partial F / \partial z)_{t}$ has a discontinuity along the line $z=0$ for $t<0$ (coexistence curve).

(3) $F$ has no singularity in $t$ along the line $t=0$ and $z \neq 0$ (critical isotherm) nor anywhere else in a neighbourhood $\left(0<z<z_{0}, 0<t<t_{0}\right)$ of the critical point.

The elementary analytical function which has the above properties is a single valued analytic function defined on the Riemann surface of an algebraic function. The algebraic function $\mathrm{f}(z, t)$ has the following characteristics.

(1) $\mathrm{f}$ has a branch point in $z$ and $t$ separately at $z=t=0$.

(2) The two smallest real branches of $f$ form a crunode (crossing point) in the $z$-plane along the line $z=0$ for $t<0$.

(3) The sheets of $f$ contain branch points whose trajectories in the $z-t$ manifold pass through the origin $(z=t=0)$. That part of the Riemann surface of the true thermodynamic potential which is relevant in the neighbourhood of the critical point is given by the Riemann surface of $f$. 
None of the other sheets of the true thermodynamic potential affect the critical exponents.

This last characteristic of $\mathrm{f}$ is illustrated by the molecular field theory. The equation of state $\tanh [(H+M) / k T]=M$ has an infinite number of solutions (sheets of $M$ as a function of $H$ ) but only the three sheets which contain the two branch points (cusps) which come together at the origin determine the critical behaviour. Thus, the reievant part of the Riemann surface for the molecular field is given by the Riemann surface of the third order algebraic function of Section VI. This Riemann surface is seen to be connected in the $H$-plane for $t \neq 0$ (the defining polynomial is irreducible in $H$ for $t \neq 0$ ) but becomes disjoint when $t=0$ (the polynomial factorizes when $t=0$ ).

The property of convexity of the thermodynamic potential has been left out of the above list since it plays no direct role in determining the qualitative properties of the Riemann surface. It is, rather, a feature which must be taken into account when constructing specific examples of thermodynamic potentiais and will, of course, play a role in determining the relationships among the critical exponents, the so-called scaling laws.

\section{REFERENCES}

1 C. de la Tour, Ann. Chim. (Phys.), [2], 21, 127 (1822).

2 T. Andrews, Trans. Roy. Soc. A, 178, 45 (1887).

3 J. D. van der Waals, Dissertation (Leiden), unpublished.

4 M. H. Coopersmith, Advanc. Chem. Phys. In press.

5 P. Weiss, J. Phys., France, 6, 667 (1907).

6 B. Widom, J. Chem. Phys. 43, 3898 (1965).

7 M. Kac, G. E. Uhlenback and P. C. Hemmer, J. Math. Phys. 4, 216 (1963).

8 L. P. Kadanoff, Physics (N.Y.), 2, 263 (1966).

9 M. H. Coopersmith, Phys. Rev. 172, 230 (1968).

${ }^{10}$ L. Onsager, Phys. Rev. 65, 117 (1944).

11 C. N. Yang, Phys. Rev. 85, 808 (1952).

12 C. Domb and D. L. Hunter, Proc. Phys. Soc. 86, 1147 (1965).

13 G. A. Baker, H. E. Gilbert, J. Eve and G. S. Rushbrooke, Physics Letters, 22, 269 (1966).

14 A. F. Andreev, Soviet Phys. JETP, 18, 1415 (1964).

15 J. S. Langer, Ann. Phys. (N.Y.), 41, 108 (1967).

${ }^{16}$ M. E. Fisher, Physics (N.Y.), 3, 255 (1967).

17 S. F. Streeter and J. E. Mayer, J. Chem. Phys. 7, 1025 (1939).

18 G. A. Bliss, Algebraic Functions, p 29. Dover: New York (1966).

19 J. S. Rowlinson, Liquids and Liquid Mixtures, p 205. Butterworths: London (1959).

20 J. T. Ho and J. D. Litster, Phys. Rev. Letters, 22, 603 (1969).

21 M. H. Coopersmith, Phys. Letters, 30A, 192 (1969). 